Methods We searched the WHO website for GRC-approved guidelines published between 2008 and November 2012. Two individuals independently appraised the guidelines using AGREE II. Scores were standardised across six domains and overall quality was determined through consensus.

Results Eighty guidelines fulfilled inclusion criteria and were appraised. Twenty-seven guidelines were recommended, 47 were recommended with modifications, and six were not recommended. Two domains of AGREE scored highly across all guidelines: scope and purpose and clarity of presentation. The rigour of development and applicability domains were variable across guidelines. The lowest scoring domains were stakeholder involvement and editorial independence.

Discussion WHO guidelines still need improvement in the following areas: stakeholder engagement, use of systematically reviewed evidence, defining the funder's role, consideration of barriers and resources (including costs) when implementing recommendations, and providing monitoring criteria. Most issues may be resolved through increased transparency and better reporting of the recommendation development process by more closely following the standards set forth in the WHO guideline development handbook.

Implications for Guideline Developers/Users Guideline developers need to ensure systematic guideline development processes are followed and adequately reported in each guideline.

\section{P133 APPROACHING ECONOMIC EVALUATION IN SOCIAL CARE GUIDANCE}

T Smith, E Shaw, N Baillie. National Institute for Health and Care Excellence, Manchester, UK

\section{0:1136/bmjqs-2013-002293.176}

Background We have a statutory responsibility to produce social care guidance. For economic evaluation challenges include: 1. Methodology for a multi-stakeholder perspective (costs and outcomes), and determining measures of effects using standardised outcomes. 2. Decision making in the absence of accepted willingness to pay thresholds, and alignment with principles used for health guidelines.

Objectives To define an economic reference case for social care guidance.

Methods A workshop on methods identified potential approaches. Health economists who work on clinical and public health guidelines were consulted to ensure consistency. Methodological issues were discussed with academic experts.

Results A reference case for social care economic evaluation was produced within a methods manual before commencing guidance development. It recognises the need for flexibility as methodology develops.

Discussion Social care economic evaluation is constrained by the quality of evidence, and the transferability of studies. Equity considerations in the context of means-tested service provision, and the issue of unpaid care, represent examples of how decision making on cost-effectiveness must take account of factors not usually considered for clinical and public health guidelines.

Implications for Guideline Developers/Users Consistent decision-making principles must be applied across all guidance development programmes, including social care cost-effectiveness. Social care guidance, developers need to recognise and work within the context of emerging methodologies when undertaking social care economic evaluation in, but ensure that such evaluations remain in line with general principles of guidance development and decision making.

\section{P135 USING CURRENT PRACTICE INFORMATION TO IDENTIFY AREAS OF VARIATION}

T Lacey, L Ayiku, E Shaw,N Baillie. National Institute for Health and Care Excellence, Manchester, UK

\section{0:1136/bmjqs-2013-002293.177}

Background Quality standards describe high-priority areas for quality improvement in a defined area.

Objectives To describe the processes by which areas for quality improvement are identified for quality standards.

Methods A topic overview, which describes core elements of the standard, such as the population and condition or services to be covered, is published on our website at the beginning of development. We then request written submissions from specialists and registered stakeholders asking them to i identify key areas for quality improvement ii provide examples of published information on current practice (such as, reports of variation in care, evaluations of guidance compliance, or patient experience) to support the identified areas. We also undertake a focused literature search for published current practice information (such as descriptions of practice variation) and identify national audits.

Results To date, we have undertaken at least 10 such reviews. We will present the types of information we receive, challenges (with a specific focus on quality of information and certainty of decisions made). We will also present how this information was used to identify area for improvement, and whether these decisions were valid.

Discussion We consider this a novel and practical approach to identifying improvement areas, bringing together views from a diverse audience, supplemented with published information.

Implications for Guideline Developers/Users Guideline developers could use similar methods to identify areas where evidence based recommendations could be focused, to define and guide best practice.

\section{P143 PROJET JALONS: A PROVINCIAL ADAPTATION OF CLINICAL PRACTICE GUIDELINES FOR DEPRESSION IN PRIMARY CARE}

${ }^{1,2} \mathrm{P}$ Roberge, ${ }^{2,3} \mathrm{~L}$ Fournier, ${ }^{2} \mathrm{H}$ Brouillet. ${ }^{1}$ University of Sherbrooke, Sherbrooke, Canada; ${ }^{2}$ Institut national de santé publique du Québec, Montréal, Canada; ${ }^{3}$ CRCHUM, University of Montreal, Montréal, Canada

\section{0:1136/bmjqs-2013-002293.178}

Background The development of a care protocol for major depression in primary care emerged as an extension of a knowledge application programme developed in Quebec (Canada) to improve care for anxiety and depressive disorders in primary care (2012; JALONS: http://www.qualaxia.org/ms/jalons/). The main goal of the project was to develop or adapt tools to support primary mental health care providers in their clinical practice.

Context The 2005 reform in Quebec's mental health services aimed at strengthening primary care services, and included the creation of multidisciplinary community-based primary mental healthcare teams. 